\title{
A stabilized mixed implicit Material Point Method for non-linear incompressible solid mechanics
}

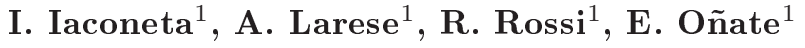 \\ International Center for Numerical Methods in Engineering (CIMNE), Technical \\ University of Catalonia (UPC) \\ Tel.: +34-93-4010808 \\ Fax: $+34-93-4016517$ \\ e-mail: iiaconeta@cimne.upc.edu, antoldt@cimne.upc.edu, rrossi@cimne.upc.edu, \\ onate@cimne.upc.edu
}

Received: date / Revised version: date

\begin{abstract}
In this work a stabilized mixed formulation for the solution of non-linear solid mechanics problems in nearly-incompressible conditions is presented. In order to deal with high material deformation, an implicit Material Point Method (MPM) is chosen. Such choice allows avoiding the classical limitations of the Finite Element Method (FEM), e.g., element tangling and extreme mesh distortion. The proposed mixed formulation, with displacement and pressure as primary variables, is tested through classical benchmarks in solid and geo-mechanics where a Neo-Hookean, a J2 and a Mohr-Coulomb plastic law are employed. Further, the stabilized mixed formulation is compared with a displacement-based formulation to demonstrate how the proposed approach gets better results in terms of accuracy, not only when incompressible materials are simulated, but also in the case of compressible ones.
\end{abstract}

Key words Particle Methods Nonlinear Finite Element Method implicit MPM mixed formulation

\section{Introduction}

The solution of solid mechanics problems in large displacement and large deformation regime, dealing with incompressible or nearly incompressible materials, is a topic of paramount importance in the computational mechanics community since many engineering problems present such conditions. It is well known that overly stiff numerical solutions appear when Poisson's 
ratio $\nu$ tends to 0.5 or when plastic flow is constrained by the volume conservation condition. In these cases, a standard Galerkin displacement-based formulation ( $\boldsymbol{u}$ formulation) fails [1,2] due to the inability to evaluate the correct strain field. In the literature, many possible solutions can be found. For instance, Simo and Rifai introduced the Mixed Enhanced Element for small deformation problems [3]. This is a special three-field mixed finite element method in which the space of discrete strains is augmented with local functions. It is worth mentioning that also the class of B-bar methods [4] and the classical incompatible modes formulation [5] fall under this theory. For general purposes, some variants of this procedure are analysed in [6]. Alternative procedures suitable for geometrically non-linear regimes, are given by the F-BAR method [7], a technique based on the concept of multiplicative deviatoric/volumetric split in conjunction with the replacement of the compatible deformation gradient field, the non-linear B-bar method [8] and the family of enhanced elements [9], which represents an extension to the non-linear regime of the procedures exposed in [4] and [5], respectively. Though the good performance of all the aforementioned methods, none of such techniques is, however, suitable for application on simplicial meshes [2,10,11. In this regards, among the successful strategies for the fulfillment of the incompressibility constraint, it is worth mentioning the group of the Mixed Variational Methods. Different researchers worked on mixed finite element formulations with displacement and mean stress as primary variables [12 16]; Cervera and coworkers, for instance, proposed a strain/displacement mixed formulation in the context of compressible and incompressible plasticity [17, 18]; Simo et al. introduced a non linear version of a three-field Hu-Washizu Variational principle, where displacement, pressure and the Jacobian of the deformation gradient are independent field variables [19]. The use of Mixed Variational Methods and the difficulties encountered when applying them with different elements have been largely discussed in the 1970s. In [20 23] the need to satisfy the stability condition, the so-called inf-sup condition, is demonstrated and the instability and ineffectiveness of elements with equal-order interpolations for all the primary variables is proved. This has motivated the development of a series of stabilization techniques, which allow the employment of low order Galerkin finite elements in computational fluid dynamics and solid mechanics problems [24]31.

The treatment of the incompressibility constraint is relatively new in the context of the Material Point Method (MPM). Most MPM formulations deal with compressible materials, avoiding the issues arising from the imposition of the incompressibility constraint. However, some procedures for the treatment of locking issues can be found in the literature. For instance, in [32 an approach for the solution of kinematic (shearing and volumetric) locking is proposed. The authors identified the employment of linear shape functions in conjunction with a regular, rectangular grid, as cause of the locking. The mixed formulation, employed in such work, is derived from the definition of a three-field Hu-Washizu potential, with stress, strain and displacement 
considered as primary variables. In 33 the formulation presented makes use of the Chorin's projection [34, a popular fractional step formulation solved implicitly for fluid mechanics problems and in [35] a similar strategy, based on a splitting operator technique for solving the momentum equation, is proposed for the treatment of the incompressibility constraint.

In this paper the computational strategy proposed in [36] for the solution of solid mechanics problems characterized by plastic incompressibility in large displacement and large deformation regime, is described in detail and applied to a wide range of test examples. A mixed $\boldsymbol{u}$ - $\boldsymbol{p}$ formulation, where the displacement and mean stress are considered as primary variables, is implemented within the framework of an implicit Material Point Method code, developed in the Kratos Multiphysics open source platform [37/38] following the algorithm presented by the authors in [39]. A monolithic solution strategy is used, which allows not to impose "spurious" pressure boundary conditions on the Neumann boundary, as done in [33, 35]. In the current work only simplicial elements are considered and a stabilization technique is adopted for the satisfaction of the inf-sup condition. The stabilization, based on the Polynomial Pressure Projection (PPP), presented in [40, is chosen for its ease of implementation and good performance demonstrated in previous works [41,42]. The proposed approach is validated through a series of benchmark examples, where an elastic Neo-Hookean, a J2 and a Mohr-Coulomb plastic material are employed. Further, for each test, the results obtained through a displacement-based $(\boldsymbol{u})$ and the stabilized mixed $(\boldsymbol{u}-p)$ formulation are compared.

The paper is organized as follow: in Section 2 the $\boldsymbol{u}$ and $\boldsymbol{u}-\boldsymbol{p}$ formulations are derived in their matrix form and the constitutive laws employed for the solution of the examples are briefly introduced. In Section 3 the Material Point Method is presented. In Section 4 the numerical examples are illustrated and the results are discussed. Finally, Section 5 presents the conclusions.

\section{The irreducible and mixed formulation}

In this section the displacement-based $(\boldsymbol{u})$ and mixed $(\boldsymbol{u}-\boldsymbol{p})$ formulations are briefly introduced and derived in matrix form.

\subsection{Governing equations in strong form}

Let us consider the body $\mathcal{B}$ which occupies a region $\Omega$ of the three-dimensional Euclidean space $\mathcal{E}$ with a regular boundary $\partial \Omega$ in its reference configuration. A deformation of $\mathcal{B}$ is defined by a one-to-one mapping

$$
\varphi: \Omega \rightarrow \mathcal{E}
$$

that maps each point $\boldsymbol{p}$ of the body $\mathcal{B}$ into a spatial point $\mathbf{x}$

$$
\mathbf{x}=\varphi(p)
$$


which represents the location of $\boldsymbol{p}$ in the deformed configuration of $\mathcal{B}$. The region of $\mathcal{E}$ occupied by $\mathcal{B}$ in its deformed configuration is denoted as $\varphi(\Omega)$.

The boundary value problem of finite elastostatics consists in finding a displacement field $\boldsymbol{u}: \varphi(\Omega) \rightarrow \mathcal{E}$ such that the equilibrium equations and the kinematic conditions are satisfied

$$
\left\{\begin{array}{rlr}
-\nabla \cdot \boldsymbol{\sigma} & =\boldsymbol{f} \text { in } & \varphi(\Omega) \\
\boldsymbol{\sigma} \cdot \boldsymbol{n} & =\overline{\boldsymbol{t}} \text { on } & \varphi\left(\partial \Omega_{N}\right) \\
\boldsymbol{u} & =\overline{\boldsymbol{u}} \text { on } & \varphi\left(\partial \Omega_{D}\right)
\end{array}\right.
$$

where $\boldsymbol{\sigma}$ is the Cauchy stress tensor, $\boldsymbol{f}$ denotes the body forces and $\varphi\left(\partial \Omega_{N}\right)$ and $\varphi\left(\partial \Omega_{D}\right)$ the boundaries of $\varphi(\Omega)$, where both the normal tension $(\overline{\boldsymbol{t}})$ (being $\boldsymbol{n}$ the outer normal) and the displacements $(\overline{\boldsymbol{u}})$ are prescribed.

As described in [1, the mixed formulation can be obtained expressing the system of Equations (3) in function of two primary variables: the displacement $\boldsymbol{u}$ and the mean stress $p$ by splitting the stress tensor in its volumetric and deviatoric part $\sigma^{\mathrm{dev}}$. Thus, the system can be rewritten as

$$
\left\{\begin{aligned}
-\nabla \cdot\left(\boldsymbol{\sigma}^{\mathrm{dev}}+p \mathbf{1}\right) & =\boldsymbol{f} \text { in } & \varphi(\Omega) \\
p-\left(\frac{1}{3} \mathbf{1}: \boldsymbol{\sigma}\right) & =0 \text { in } & \varphi(\Omega) \\
\left(\boldsymbol{\sigma}^{\mathrm{dev}}+p \mathbf{1}\right) \cdot \boldsymbol{n} & =\overline{\boldsymbol{t}} \text { on } & \varphi\left(\partial \Omega_{N}\right) \\
\boldsymbol{u} & =\overline{\boldsymbol{u}} \text { on } & \varphi\left(\partial \Omega_{D}\right)
\end{aligned}\right.
$$

being 1 the second order identity tensor. We can observe that if $\boldsymbol{u}$ is a solution of Equation (3), then $(\boldsymbol{u}, p)$, satisfying also $p-\left(\frac{1}{3} \boldsymbol{1}: \boldsymbol{\sigma}\right)=0$, is a solution of Equation (4).

\subsection{Weak form and linearisation of the weak form in spatial form}

Following the standard FEM procedure, the weak forms of Equations (3) and (44) are obtained by employing the Galerkin method and are written in spatial configuration, adopting an Updated Lagrangian framework.

For the displacement formulation, the first equation of (3) is multiplied by a test function $\boldsymbol{w}$, which is lying in the space $\mathcal{V}$ of kinematically admissible displacements, such that $\boldsymbol{w}=\left\{\boldsymbol{w} \in \mathcal{V} \mid \boldsymbol{w}=\mathbf{0}\right.$ on $\left.\varphi\left(\partial \Omega_{D}\right)\right\}$. By using the divergence theorem the weak form of momentum balance can be obtained as

$G_{(\boldsymbol{u})}(\boldsymbol{w})=\int_{\varphi(\Omega)} \boldsymbol{\sigma}:\left[\nabla^{s} \boldsymbol{w}\right] d v-\int_{\varphi(\Omega)} \boldsymbol{f} \cdot \boldsymbol{w} d v-\int_{\varphi\left(\partial \Omega_{N}\right)} \overline{\boldsymbol{t}} \cdot \boldsymbol{w} d a=0, \quad \forall \boldsymbol{w} \in \mathcal{V}$

using the notation $\mathbf{A}^{s}=\frac{1}{2}\left(\mathbf{A}+\mathbf{A}^{T}\right)$. In this work a Newton-Raphson's iterative procedure is employed for the solution of problems characterized by material and geometrical non-linearities. The non-linear weak form of Equation (5) has to be linearized through an expansion in Taylor's series, evaluated at the last known equilibrium configuration $\boldsymbol{u}^{*}$, expressed as

$$
L(\delta \boldsymbol{u}, \boldsymbol{w}) \simeq G_{\left(\boldsymbol{u}^{*}\right)}(\boldsymbol{w})+D_{\boldsymbol{u}} G_{\left(\boldsymbol{u}^{*}\right)}(\boldsymbol{w})[\delta \boldsymbol{u}]=0, \quad \forall \boldsymbol{w} \in \mathcal{V}
$$


where $L$ is the linearised virtual work and $D_{\boldsymbol{u}} G_{\left(\boldsymbol{u}^{*}\right)}(\boldsymbol{w})[\delta \boldsymbol{u}]$ is the directional derivative of $G$ at $\boldsymbol{u}^{*}$ in the direction of $\delta \boldsymbol{u}$. Assuming that only internal force contributions are dependent on the displacement primary variable, the directional derivative can be written as

$$
D_{\boldsymbol{u}} G_{\left(\boldsymbol{u}^{*}\right)}(\boldsymbol{w})[\delta \boldsymbol{u}]=\left.\frac{d}{d \gamma}\right|_{\gamma=0}\left(\int_{\varphi(\Omega)}\left[\boldsymbol{\sigma}(\boldsymbol{\epsilon}(\gamma)):\left(\nabla^{s} \boldsymbol{w}\right)\right] d v\right)
$$

where $\gamma$ is a scalar parameter and the following expression is used

$$
\boldsymbol{\epsilon}(\gamma)=\nabla^{s}\left(\boldsymbol{u}^{*}+\gamma \delta \boldsymbol{u}\right)=\boldsymbol{\epsilon}^{*}+\gamma \nabla^{s}(\delta \boldsymbol{u}) .
$$

with $\epsilon^{*}=\nabla^{s}\left(\boldsymbol{u}^{*}\right)$ the strain field at $\boldsymbol{u}^{*}$. The final matrix form can be obtained as

$$
\mathbf{K}^{\tan } \delta \boldsymbol{u}=-\mathbf{R}
$$

where $\delta \boldsymbol{u}$ is the vector of unknowns, $\mathbf{R}$ is the vector of residuals, expressed as

$$
\mathbf{R}=G_{\left(\boldsymbol{u}^{*}\right)}(\boldsymbol{w})
$$

and $\mathbf{K}^{\tan }=D_{\boldsymbol{u}} G_{(\boldsymbol{u})}(\boldsymbol{w})$ is the tangent matrix, evaluated as the sum of the material stiffness matrix

$$
\mathbf{K}^{M}:=\int_{\varphi(\Omega)}\left[\nabla^{s} \boldsymbol{w}\right] \mathbf{D}\left[\nabla^{s} \delta \boldsymbol{u}\right] d v
$$

and the geometric stiffness matrix

$$
\mathbf{K}^{G}:=\int_{\varphi(\Omega)}[\nabla \boldsymbol{w}] \boldsymbol{\sigma}[\nabla \delta \boldsymbol{u}] d v
$$

where $\mathbf{D}$ is the spatial algorithmic tangent moduli. The detailed procedure to derive the final expression of the system of linearised equations in integral and discrete form can be found in [39] and [43].

Concerning the mixed formulation, linear interpolation finite elements both for displacement and pressure $(\boldsymbol{u}-p)$ are considered. In this regard, the weak form of the balance of the linear momentum (Equation (5) can be rewritten as

$$
\begin{array}{r}
G_{(\boldsymbol{u}, p)}(\boldsymbol{w})=\int_{\varphi(\Omega)}\left(\boldsymbol{\sigma}^{\mathrm{dev}}+p \mathbf{1}\right):\left[\nabla^{s} \boldsymbol{w}\right] d v-\int_{\varphi(\Omega)} \boldsymbol{f} \cdot \boldsymbol{w} d v- \\
\int_{\varphi\left(\partial \Omega_{N}\right)} \overline{\boldsymbol{t}} \cdot \boldsymbol{w} d a=0, \quad \forall \boldsymbol{w} \in \mathcal{V}
\end{array}
$$

where the Cauchy stress tensor $\sigma$ is decomposed in its deviatoric and volumetric component, denoted as $\boldsymbol{\sigma}^{\mathrm{dev}}$ and $p$, respectively. The weak form of the pressure continuity equation is obtained by performing a $L_{2}$ inner product of the second equation of (4) with an arbitrary test function $q \in \mathcal{Q}$, 
where $\mathcal{Q}$ is the space of virtual pressure. Finally the weak form of the pressure continuity equation is expressed as

$$
G_{(\boldsymbol{u}, p)}(q)=\int_{\varphi(\Omega)} q\left[\left(\frac{1}{3} \mathbf{1}: \boldsymbol{\sigma}\right)-p\right] d v=0, \quad \forall q \in \mathcal{Q}
$$

By performing a linearization of Equations (13) and (14), similarly to what explained for Equation (6), the solution system of linearized equations can be derived and expressed in matrix form as

$$
\left[\begin{array}{cc}
{ }^{m} \mathbf{K}^{\tan } & \mathbf{B} \\
\mathbf{B}^{*} & -\mathbf{M}
\end{array}\right]\left[\begin{array}{l}
\delta \boldsymbol{u} \\
\delta \boldsymbol{p}
\end{array}\right]=-\left[\begin{array}{l}
\boldsymbol{R}_{\boldsymbol{u}} \\
\boldsymbol{R}_{p}
\end{array}\right]
$$

where $\mathbf{R}_{u}=G_{(\boldsymbol{u}, p)}(\boldsymbol{w})$ and $\boldsymbol{R}_{p}=G_{(\boldsymbol{u}, \boldsymbol{p})}(q)$ are the components of the residual vector, $\delta \boldsymbol{u}$ and $\delta \boldsymbol{p}$ are the vector of unknown displacements and unknown mean stresses, respectively. The components of the matrix on the left hand side (lhs) of Equation (15) are given by the tangent stiffness matrix ${ }^{m} \mathbf{K}^{\tan }=D_{\boldsymbol{u}} G_{(\boldsymbol{u}, p)}(\boldsymbol{w})$, which can be seen as the sum of the material stiffness matrix

$$
{ }^{m} \mathbf{K}^{M}:=\int_{\varphi(\Omega)}\left[\nabla^{s} \boldsymbol{w}\right]\left(\mathbf{D}^{\mathrm{dev}}+p(\mathbf{1} \otimes \mathbf{1}-2 \llbracket)\right)\left[\nabla^{s} \delta \boldsymbol{u}\right] d v
$$

being $\llbracket$ the fourth order identity tensor, and the geometric stiffness matrix

$$
{ }^{m} \mathbf{K}^{G}:=\int_{\varphi(\Omega)}[\nabla \boldsymbol{w}]\left(\boldsymbol{\sigma}^{\mathrm{dev}}+p \mathbf{1}\right)[\nabla \delta \boldsymbol{u}] d v
$$

Furthermore, $\mathbf{M}=D_{p} G_{(\boldsymbol{u}, p)}(q)$ is

$$
\mathbf{M}=\int_{\varphi(\Omega)} q \delta p d v
$$

and the mixed terms $\mathbf{B}=D_{p} G_{(\boldsymbol{u}, p)}(\boldsymbol{w})$ and $\mathbf{B}^{*}=D_{\boldsymbol{u}} G_{(\boldsymbol{u}, p)}(q)$, are defined, respectively, as

$$
\begin{gathered}
\mathbf{B}=\int_{\varphi(\Omega)}(\nabla \cdot \boldsymbol{w}) \delta p d v \\
\mathbf{B}^{*}=\int_{\varphi(\Omega)} D_{\boldsymbol{u}}\left(\frac{1}{3} \mathbf{1}: \boldsymbol{\sigma}\right)(\nabla \cdot \delta \boldsymbol{u}) q d v
\end{gathered}
$$

where $D_{u}\left(\frac{1}{3} 1: \sigma\right)$ can be derived once determined the volumetric stress as function of the strain field.

One can observe that ${ }^{m} \mathbf{K}^{M}$ and ${ }^{m} \mathbf{K}^{G}$ are distinguished from $\mathbf{K}^{M}$ and $\mathbf{K}^{G}$, defined for the irreducible formulation (Equations (11) and (12)). In the mixed case, the deviatoric part of $\mathbf{D}$ and $\sigma$ is separated by the volumetric one and an evaluation of the latter is done, not using the material response of the constitutive law, but the interpolation of the nodal pressure field on the material points, i.e., the integration points. 
For the treatment of the incompressibility constraint, the Polynomial Pressure Projection (PPP), introduced by Dohrmann and Bochev [40], is used. This stabilization procedure is obtained by modifying the mixed variational equation by using a $L^{2}$ polynomial pressure projection. If $k$ is the order of the continuous polynomial shape functions used to approximate $p$, the pressure projection is performed into a polynomial space with order of $k-1$. As in the current work linear shape functions are used for the pressure, the $L^{2}$ polynomial pressure projection is made in a discontinuous space and, consequently, it can be performed at the element level as

$$
\int_{\varphi(\Omega)} \tilde{q}(p-\tilde{p}) d v=0, \quad \forall \tilde{q} \in \mathcal{Q}^{0}
$$

being $\tilde{p}$ the best approximation of $p$ in $\left(\mathcal{Q}^{0}\right)$ and $\tilde{q} \in \mathcal{Q}^{0}$ an arbitrary test function, where $\mathcal{Q}^{0}$ is the space of polynomial functions with zero degree in each coordinate direction. Unlike other stabilization techniques, the pressure stabilization is accomplished without the use of the residual of the momentum equation; thus the calculation of higher-order derivatives and the specification of a mesh-dependent stabilization parameter are avoided. Moreover, it is demonstrated that symmetry of the mixed formulation is retained.

In the case of simplicial elements, as in the current work, the stabilization of the unstable mixed formulation requires only the addition of the bilinear form

$$
\int_{\varphi(\Omega)^{e}}(q-\tilde{q}) \frac{\alpha}{G}(p-\tilde{p}) d v=0
$$

to Equation (14), where $\alpha$ is a parameter to be selected for stability and $G$ the shear modulus. The weak form of the pressure continuity equation (Equation (14) ) can be rewritten as

$$
G_{(\boldsymbol{u}, p)}(q)=\int_{\varphi(\Omega)} q\left[\left(\frac{1}{3} \mathbf{1}: \boldsymbol{\sigma}\right)-p\right]-\frac{\alpha}{G}[q p-\tilde{q} \tilde{p}] d v=0
$$

and the matrix system (Equation (15)) becomes

$$
\left[\begin{array}{cc}
\mathbf{K}^{\tan } & \mathbf{B} \\
\mathbf{B}^{*} & -\mathbf{M}-\mathbf{M}^{\text {stab }}
\end{array}\right]\left[\begin{array}{l}
\delta \boldsymbol{u} \\
\delta \boldsymbol{p}
\end{array}\right]=-\left[\begin{array}{c}
\boldsymbol{R}_{u} \\
\boldsymbol{R}_{p}+\boldsymbol{R}_{p}^{\text {stab }}
\end{array}\right]
$$

where

$$
\mathbf{M}^{\mathrm{stab}}=\int_{\varphi(\Omega)} \frac{\alpha}{G}(q \delta p-\tilde{q} \delta \tilde{p}) d v
$$

and

$$
R_{p}^{\mathrm{stab}}=\int_{\varphi(\Omega)} \frac{\alpha}{G}[q p-\tilde{q} \tilde{p}] d v
$$




\subsection{Constitutive models}

In this section the constitutive laws, employed in the current work, are briefly discussed. In Section 4, a hyperelastic Neo-Hookean law, a hyperelasticplastic J2 and Mohr-Coulomb plastic laws in finite strains are employed.

2.3.1 Hyperelastic law The hyperelastic law, used in Section 4.1, is a NeoHookean model with a stored energy function $W$ written as the sum of its volumetric $U(J)$ and deviatoric $\tilde{W}(\bar{b})$ component

$$
W(J, \overline{\boldsymbol{b}}):=K U(J)+\tilde{W}(\overline{\boldsymbol{b}})=\frac{1}{2} K(J-1)^{2}+\frac{1}{2} G(\operatorname{tr}(\overline{\boldsymbol{b}})-3)
$$

with $K$ representing the bulk modulus, $J=\operatorname{det}(\boldsymbol{F})$ the determinant of the total deformation gradient and $\bar{b}$ the volume preserving part of the left Cauchy-Green tensor $\boldsymbol{b}=\boldsymbol{F} \boldsymbol{F}^{T}$, defined as

$$
\bar{b}:=J^{-\frac{2}{3}} \boldsymbol{b}
$$

The Kirchhoff stress $\tau$ can be defined as

$$
\boldsymbol{\tau}:=K J(J-1)+G \operatorname{dev}(\bar{b})
$$

and in the case of the mixed formulation, Equation (29) is reformulated as

$$
\boldsymbol{\tau}=p_{p} J \mathbf{1}+G \operatorname{dev}(\overline{\boldsymbol{b}})
$$

where $p_{p}$ is the pressure value on the material point.

2.3.2 Hyperelastic - J2 plastic law Regarding the hyperelastic-plastic J2 law employed in Section 4.2, the approach presented in [44, based on the multiplicative decomposition of $\boldsymbol{F}$, is adopted. Following this approach, $\boldsymbol{F}$ is split into a plastic and elastic contribution as

$$
\boldsymbol{F}=\boldsymbol{F}^{e} \boldsymbol{F}^{p}
$$

For the evaluation of the material response in the elastic regime, the reader has to refer to the stored energy function (Equation (27)), while for the plastic regime, the system of equations, represented by the yield surface $f(\boldsymbol{\tau}, q)$, the plastic flow rule $\left(\dot{\boldsymbol{C}}^{p}\right)^{-1}$ deduced by [45] for associative $J_{2^{-}}$ plasticity, with the Kuhn-Tucker conditions, has to be considered:

$$
\left\{\begin{array}{l}
f(\boldsymbol{\tau}, q)=|\operatorname{dev}(\boldsymbol{\tau})|-\sqrt{\frac{2}{3}} \boldsymbol{\sigma}_{Y} \leq 0 \\
\left(\overline{\boldsymbol{C}^{p}}\right)^{-1}=-\frac{2}{3} \dot{\lambda} \operatorname{tr}\left(\boldsymbol{b}^{e}\right) \boldsymbol{F}^{-1} \boldsymbol{n} \boldsymbol{F}^{-T}
\end{array}\right.
$$

where $\dot{\boldsymbol{C}^{p}}$ is the inverse of the volume preserving part of the plastic right Cauchy-Green deformation tensor $\overline{\boldsymbol{C}^{p}}={\overline{\boldsymbol{F}^{p}}}^{T} \overline{\boldsymbol{F}^{p}}, \lambda$ is the plastic multiplier, $\boldsymbol{n}$ is the unit vector of $\operatorname{dev}(\boldsymbol{\tau})$ and $\boldsymbol{\sigma}_{Y}$ is the yield stress. As a perfectly plastic law is used, the hardening law is neglected. 
2.3.3 Hyperelastic - Mohr-Coulomb plastic law In Section 4.3 a MohrCoulomb plastic law is considered. For its implementation the implicit integration scheme in principal stress space, presented in [46], is followed. Also in this case no hardening law is used and in the formulation it will be neglected. For the evaluation of the elasto-plastic response the system of equations, represented by the yield surface $f(\boldsymbol{\sigma})$, the plastic flow rule $\dot{\boldsymbol{\epsilon}}^{p}$, with the Kuhn-Tucker conditions, is

$$
\left\{\begin{array}{l}
f\left(\sigma_{1}, \sigma_{2}, \sigma_{3}\right)=\left(\sigma_{1}-\sigma_{3}\right)+\left(\sigma_{1}+\sigma_{3}\right) \sin \phi-2 c \cos \phi \leq 0 \\
\dot{\boldsymbol{\epsilon}}^{p}=\dot{\lambda} \frac{\partial g(\boldsymbol{\sigma})}{\partial \boldsymbol{\sigma}}
\end{array}\right.
$$

where $\sigma_{1}>\sigma_{2}>\sigma_{3}$ are the major and minor principal stresses, $\phi$ is the angle of internal friction and $c$ the cohesion. As in the current work an associative flow rule is considered, the plastic potential $g(\boldsymbol{\sigma})$ coincides with the yield surface function $f(\boldsymbol{\sigma})$, i.e., $g(\boldsymbol{\sigma})=f(\boldsymbol{\sigma})$.

According to [47,48], it is possible to redefine the scheme of the algorithm presented in [46] for a finite strain regime, considering the Hencky strain measure, evaluated in principal space as

$$
\boldsymbol{\epsilon}=\frac{1}{2} \ln (\boldsymbol{\beta})
$$

where $\boldsymbol{\beta}$ is the eigenvalue vector of the elastic left Cauchy-Green deformation tensor $\boldsymbol{b}^{e}$, whose trial value is given by

$$
\boldsymbol{b}^{e, \text { trial }}=\Delta \boldsymbol{F} \cdot \boldsymbol{b}^{e, n} \cdot \Delta \boldsymbol{F}^{T}
$$

where $\boldsymbol{b}^{e, n}$ is the converged value relative to the last known configuration at time $t^{n}$ and $\Delta \boldsymbol{F}:=\frac{\partial \varphi\left(\boldsymbol{X}, t^{n+1}\right)}{\partial \varphi\left(\boldsymbol{X}, t^{n}\right)}$ is the increment of the total deformation gradient between $t^{n}$ and $t^{n+1}$. The free energy function is a quadratic function of the principal elastic Hencky strains $\left(\epsilon_{1}^{e}, \epsilon_{2}^{e}, \epsilon_{3}^{e}\right)$ [4]

$$
\psi\left(\boldsymbol{\epsilon}_{A}^{e}\right)=\frac{1}{2} \lambda\left[\epsilon_{1}^{e}+\epsilon_{2}^{e}+\epsilon_{3}^{e}\right]^{2}+\mu\left[\left(\epsilon_{1}^{e}\right)^{2}+\left(\epsilon_{2}^{e}\right)^{2}+\left(\epsilon_{3}^{e}\right)^{2}\right]
$$

with $\lambda$ and $\mu$ denoting the Lamé constants and $\epsilon_{A}^{e}$ the elastic Hencky strains along the three principal directions.

\section{The Material Point Method}

The Material Point Method (MPM) is a particle-based method, whose origin goes back to the work of Harlow [49], who proposed the particle-in-cell method (PIC), as a technique for the solution of fluid flow problems. Some decades after, in the works of Sulsky and coworkers [50,51, the PIC method was redefined within the solid mechanics framework, adopting the name of Material Point Method. MPM combines the use of a Lagrangian description of the continuum, represented by the material points, with a discretization 
of the computational domain, given, in this case, by an Eulerian grid, as can be observed in Figure 1. This distinctive feature allows to track the deformation of the body and retrieve the history-dependent material information at each time instant of the simulation, without committing the state variables mapping errors, typical of methods, which make use of remeshing techniques. This makes the method particularly attractive for the solution of problems, characterized by very large deformations and by the use of complex constitutive laws 52,53 .

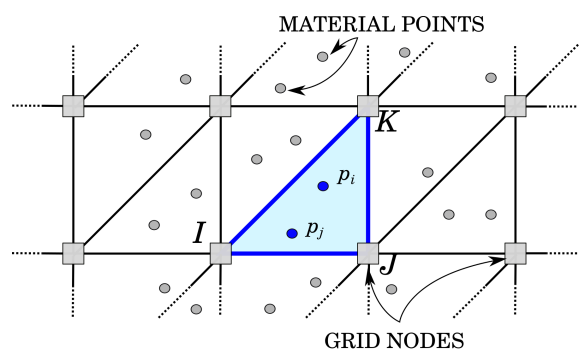

Figure 1 MPM: Overlapping of material points and Eulerian grid (source: 39]).

Since the works published in 50,51, many improvements have been provided for a more efficient and algorithmically straightforward evaluation of grid node integrals in the weak formulation, such as, the generalized interpolation material point method (GIMP) [54], the convected particle domain interpolation technique (CPDI) [55] or the second-order convected particle domain interpolation (CPDI2) [56]. In these versions, unlike the original MPM, the particle mass is smeared over a particle domain (area or volume) defined by an undeformable or a deformable parallelogram or a deformable quadrilateral, in GIMP, CPDI and CPDI2, respectively. Alternative techniques, which attempt to improve the nodal shape functions base, make use of higher order interpolation functions [57-59].

The MPM formulation, in the current work, uses the classical interpolation procedure with linear shape functions and an implicit Newmark time integration scheme. Such approach has been developed for taking into account of both geometric and material non-linearities; for this reason the non-linear governing equations have to be linearized and solved through an iterative Newton-Raphson scheme. Extension to GIMP, CPDI or CPDI2 versions can also be considered, but goes out of the scope of the present work, where a mixed MPM formulation is presented.

As in the classic MPM, the algorithm consists of three main steps (Figure 2):

(a) Initialization phase: at the beginning of the calculation step, the initial nodal conditions are evaluated through a mapping of the material point information on the nodes of the computational grid (Figure 2(a)); 
(b) UL FEM phase: once the initial conditions on the nodes are created, it is possible to evaluate the nodal unknowns in terms of displacement and pressure (Figure 2(b);

(c) Convective phase: as last step, the material point information are updated throughout an interpolation of the nodal data. Before concluding the current time step, the material points are moved to a new position and the nodal information are deleted (Figure 2(c)].

For further details, the algorithm, followed for the implementation of the MPM code, can be found in [39].

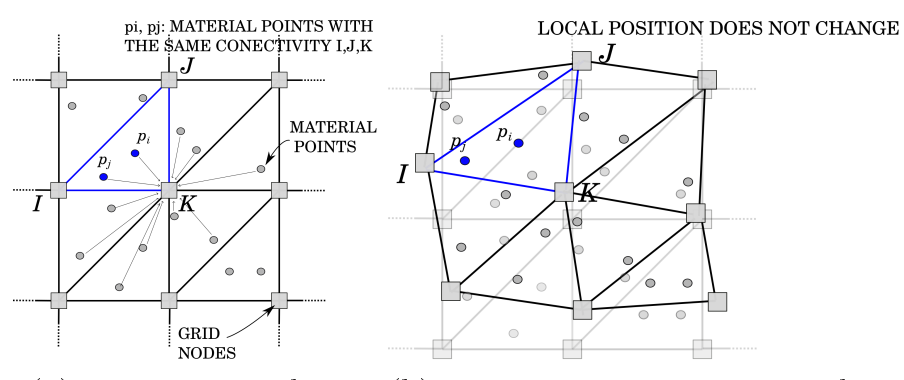

(a) Initialization phase (b) Updated Lagrangian FEM phase

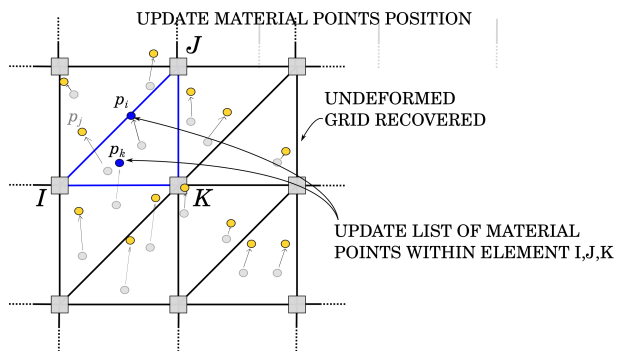

(c) Convective phase

Figure 2 MPM phases (source: [39]).

If a mixed $(\boldsymbol{u}-p)$ formulation is used in the framework of the MPM, it is important to highlight that some changes have to be considered in the initialization and convective phase, described in 39. In the initialization phase, initial nodal pressure values $p_{I}^{n}$, related to the previous time $t^{n}$, have to be evaluated, in addition to the mass, velocity and acceleration ones, using the following expression:

$$
p_{I}^{n}=\frac{\sum_{p} N_{I} m_{p} p_{p}^{n}}{\sum_{p} N_{I} m_{p}}
$$

where $N_{I}$ is the shape function of node $I$ evaluated at the position of the $p$ - th material point, and $m_{p}$ and $p_{p}^{n}$ are the mass and the pressure of the material point, respectively. The nodal pressure evaluated in Equation (37) 
is used in the predictor step of the Newmark scheme. Once the solution is iteratively computed using the linearized system of Equations (24), the convective phase is performed, as explained in detail in [39. The pressure on the material points is updated in addition to the material point displacement, velocity and acceleration, through an interpolation of the current nodal pressure values $p_{I}^{n+1}$ on the material point position

$$
p_{p}^{n+1}=\sum_{I} N_{I} p_{I}^{n+1} .
$$

\section{Numerical Examples}

In this section, three numerical examples are presented for the validation of the mixed formulation. Firstly, the well-known benchmark test of a Cook's elastic membrane is considered and a mesh convergence study is performed. The stability of the mixed formulation is assessed in a quasi-incompressible elastic case. Secondly, a plane strain tension test of a J2-plastic plate in compressible and incompressible state is analysed. In this example, the performances of the irreducible $\boldsymbol{u}$ and the mixed $\boldsymbol{u}-\boldsymbol{p}$ formulations are compared in the case of incompressible plastic flow. Finally, a plain strain rigid strip footing is studied. The reason for choosing such example is twofold: on one hand it represents a case of saturated porous solid in undrained conditions which is known to suffer from volumetric locking. On the other hand, the MPM formulation can be validated in large displacement and large deformation regimes. The results obtained with the $\boldsymbol{u}$ and $\boldsymbol{u}$ - $p$ formulations are compared and used to demonstrate that a mixed MPM formulation can provide more accurate and reliable results, not only under the assumption of elastic and plastic incompressibility, but even in compressible situations.

In this work a stabilization parameter $(\alpha)$ with value of 1 has been used. The direct solver SuperLU is employed for the solution of the system of linearized equations, both in the case of $\boldsymbol{u}$ and $\boldsymbol{u}-\boldsymbol{p}$ formulations.

\subsection{Cook's membrane problem}

As a first numerical example, we consider the well known Cook's membrane test, proposed for the first time by Cook [60]. This test is often used as a benchmark to check the element formulation under compressible and incompressible conditions. In the literature, the Cook's membrane is commonly tested in infinitesimal deformation assumption and material linearity [14, geometric non-linearity and material linearity [61] and, finally, in geometric and material non-linearities [7, 13, 41,47]. The geometry and material properties of the problem are shown in Figure 3. A clamped trapezoidal plate, subjected to a distributed shear load, whose resultant force is $P=1 N$, applied along the right side, is analysed. The static case is solved studying the response of a compressible and a quasi-incompressible Neo-Hookean 
material, whose stored energy function is defined by Equation (27). The convergence study is performed using six structured triangular meshes each of which uses an initial value of one material point per element.

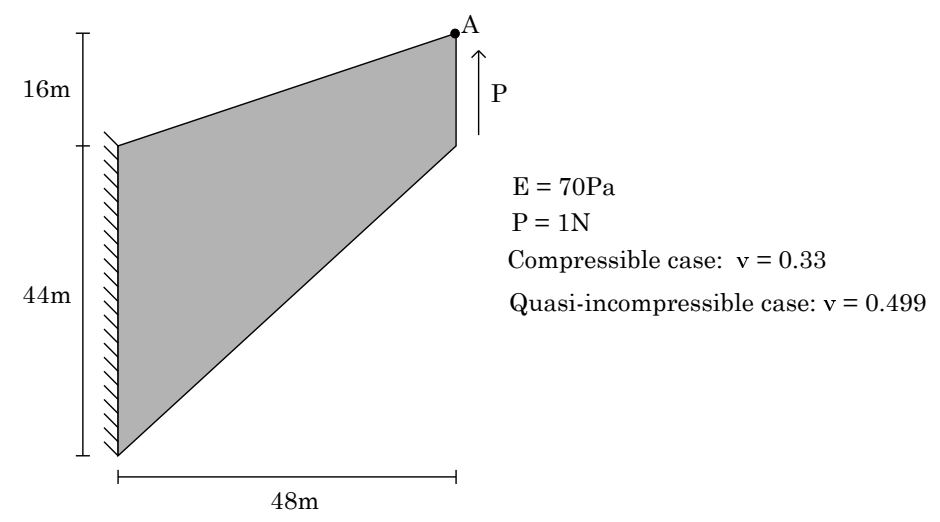

Figure 3 Cook's membrane. Geometry, material properties and boundary conditions

Since the formulations under study are based on the assumption of finite deformation and material non-linearity, the results relative to a very fine mesh (256 elements per side) of a FEM analsys is considered as reference solution in the compressible case, while the result of [41] is the benchmark solution for the quasi-incompressible case. The reference solution of vertical displacement at point A (Figure 3) is found to be $0.323 \mathrm{~m}$, in the compressible case, and $0.275 \mathrm{~m}$ in the quasi-incompressible cases, respectively. The results of $\boldsymbol{u}$ and $\boldsymbol{u}$ - $p$ formulations, with and without stabilization term (UP No Stab and UP Stab) are summarized in Table (1) for both the compressible and nearly incompressible cases. The same results can be observed graphically in Figures 4 and 5 .

Table 1 Cook's membrane. Compressible case: vertical displacement at point A obtained with the U, UP formulation without and with stabilization

\begin{tabular}{|c|ccc|crc|}
\hline Elements per side & \multicolumn{3}{|c|}{ Compressible case } & \multicolumn{3}{c|}{ Quasi-incompressible case } \\
\hline & U & UP No Stab & UP Stab & U & UP No Stab & UP Stab \\
\hline 2 & 0.089 & 0.1013 & 0.1172 & 0.0723 & 0.0788 & 0.1277 \\
\hline 4 & 0.1415 & 0.1718 & 0.1953 & 0.0736 & 0.1157 & 0.1932 \\
\hline 8 & 0.2183 & 0.2511 & 0.2669 & 0.0742 & 0.1821 & 0.2424 \\
\hline 16 & 0.2771 & 0.2952 & 0.3025 & 0.075 & 0.2356 & 0.2648 \\
\hline 32 & 0.30386 & 0.3119 & 0.315 & 0.0775 & 0.2606 & 0.2725 \\
\hline 64 & 0.3133 & 0.3176 & 0.319 & 0.0862 & 0.2702 & 0.275 \\
\hline
\end{tabular}


The $\boldsymbol{u}$ formulation is less accurate than the $\boldsymbol{u}$ - $p$ formulation both for the UP No Stab and UP Stab cases, not only for the nearly incompressible condition, as expected, but also for the compressible one. However, the discrepancy is clearly visible in the quasi-incompressible problem (Figure 5), where the capability of the $\boldsymbol{u}$ formulation to predict the displacement field is compromised due to volumetric locking.

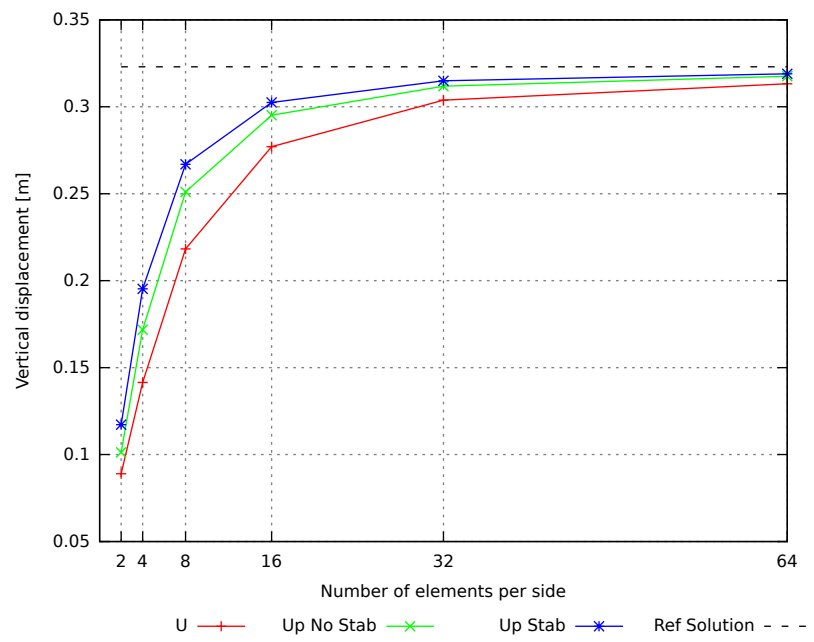

Figure 4 Cook's membrane. Compressible case: vertical displacement at point A

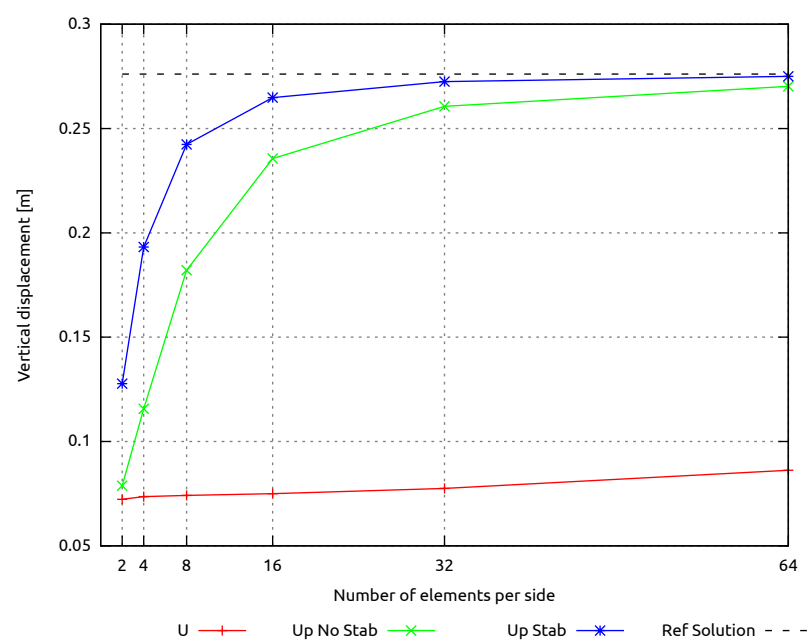

Figure 5 Cook's membrane. Quasi-incompressible case: vertical displacement at point A 
Regarding the mixed approaches, from Figure [5] it is possible to infer that even not using a stabilization term the solution is not affected by volumetric locking. However, through the stabilized $\boldsymbol{u}$ - $p$ formulation it is also possible to prevent pressure oscillation issues in the mean stress field, as can be observed in Figure 6, where the pressure values of Figure 6(a) are all out of the threshold defined by the solution of Figure 6(b).

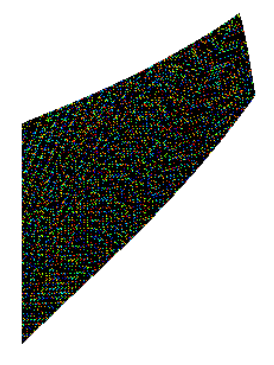

(a) $u$-p without stabilization

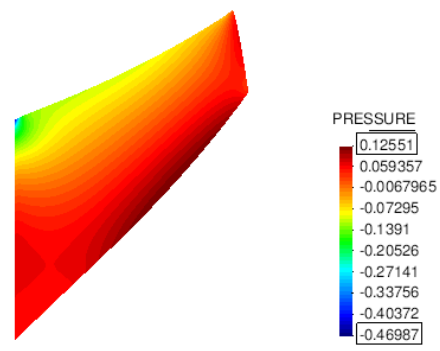

(b) $\boldsymbol{u}$ - $p$ with stabilization

Figure 6 Cook's membrane. Quasi-incompressible case: Pressure counter fill. The mixed formulation without any stabilization (a) fails to predict the pressure field, while it is correctly evaluated using the PPP stabilization (b). Black contour colour should be intended as out of range.

\subsection{D tension test}

As second numerical example, a plane strain tension problem is considered to test the mixed formulation in an elasto-plastic regime. A 2D plate, clamped at the bottom of the specimen, is subjected to a prescribed vertical displacement on the upper side. Both geometry and material properties are taken from [16] and are depicted in Figure 7 . The plate is made by a hyperelastic perfectly-plastic material which is simulated using a J2 plastic law. An unstructured triangular background mesh with a mesh size of $0.001 \mathrm{~m}$ and an initial distribution of 12 material points per cell, which is found to give the optimal trade-off between accuracy of the results and computational cost in both the compressible and incompressible cases, are adopted.

The results of the compressible case are shown in Figures 8, 9, 10 and 11. where the displacement along $\mathrm{x}$ and $\mathrm{y}$-direction, the equivalent plastic strains and the vertical Cauchy stresses are shown. Volumetric locking is not affecting the numerical results, as the plate is working under compressible conditions. However, the $\boldsymbol{u}-p$ formulation is more accurate than the $\boldsymbol{u}$ one, not only in the evaluation of the stress field, but also of the displacement field. Moreover, the goodness of the solution can be appreciated looking at Figure 10(b) the equivalent plastic strains are distinctly distributed along a cross shape, while the result of Figure 10(a) revokes the same shape, but without the same order of precision. In conclusion, even if a compressible 


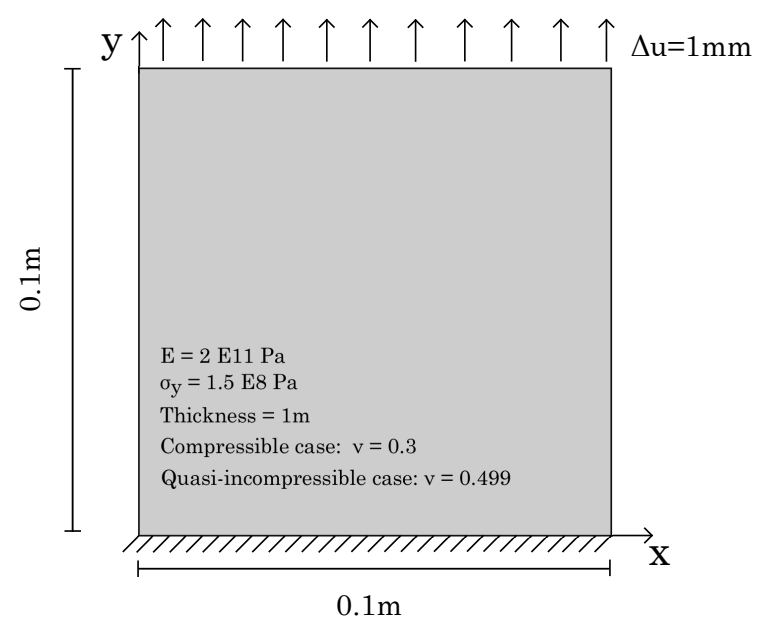

Figure 7 Tension test. Geometry, material properties and boundary conditions

material is simulated, the results obtained with the $\boldsymbol{u}$ - $p$ formulation present a higher order of accuracy, by using the same mesh size and the same number of material points per element.

The results of the incompressible case are shown in Figures 12, 13, 14, and 15. In this case, the $\boldsymbol{u}$ formulation fails in the simulation of the tension test. As expected, the displacement and stress fields are affected by volumetric locking and the plastic deformations are incorrectly localized. On the other hand, Figures 12(b), 13(b), 14(b) and 15(b) show that the $\boldsymbol{u}$ - $p$ formulation is able to evaluate correctly the displacement and stress field under incompressible conditions. The results are similar to those depicted in Figures 8(b) 9(b), 10(b) and 11(b) the cross-shape distribution of the equivalent plastic strains and stresses are recovered. Furthermore, Figures 16(a), 16(b), $16(\mathrm{c})$ and 16(d) show a comparison in the nearly-incompressible case between the reference solution obtained with the formulation proposed in 16 and the results obtained with the MPM $\boldsymbol{u}$ - $p$ formulation presented in the current work. We can observe that there is a good agreement both in the distribution of equivalent plastic strains and pressure fields and in their values range. Finally, the stress - displacement curve, evaluated with the mixed formulation, is shown in Figure 17. The results for the compressible and incompressible cases are in good agreement. Both correctly predict the elastic regime and the inception of the plastic flow when the yield stress is reached.

Since a mixed formulation with displacement and pressure as primary variables is adopted, strains are not linearly distributed within the element, but these coincide with a constant function. It worth highlighting that through this numerical procedure while it is possible to avoid the volumetric locking, the problems related with strain localization are still present. This means that the width of the shear bands still depends on the size of the 
elements. This problem can be solved by regularization of the element size as proposed, e.g. in [17,18,62, or [48, where the formulations consider the strain field as primary variable and, therefore, its linear distribution can be evaluated, which allows to accurately predict strain localization with mesh independence.

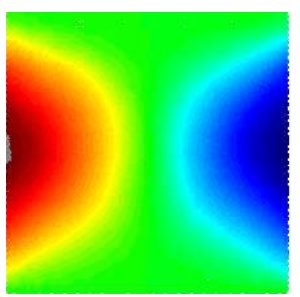

(a) $u$ formulation

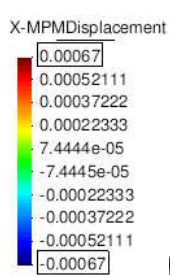

00067

Figure 8 Tension test. Compressible case: horizontal displacement

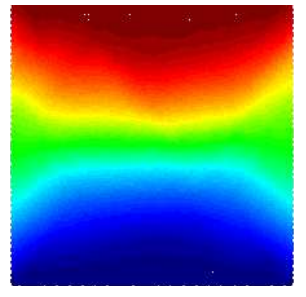

(a) $u$ formulation

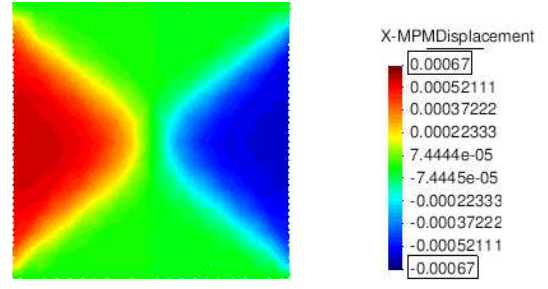

(b) $\boldsymbol{u}$ - $p$ formulation

Figure 9 Tension test. Compressible case: vertical displacement
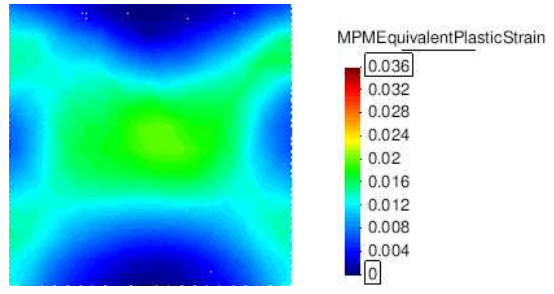

(a) $u$ formulation

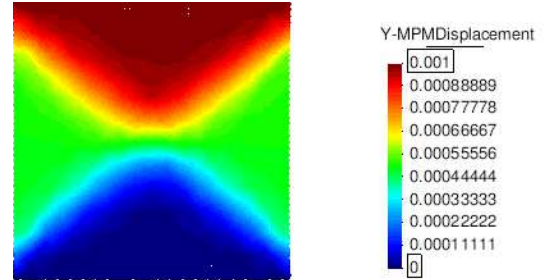

(b) $u$ - $p$ formulation

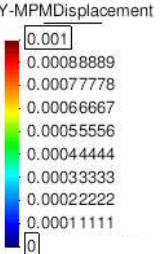

\begin{abstract}
mpressible case: vertical displacement
\end{abstract}




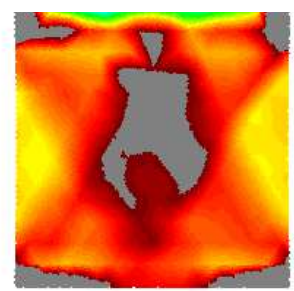

(a) $\boldsymbol{u}$ formulation
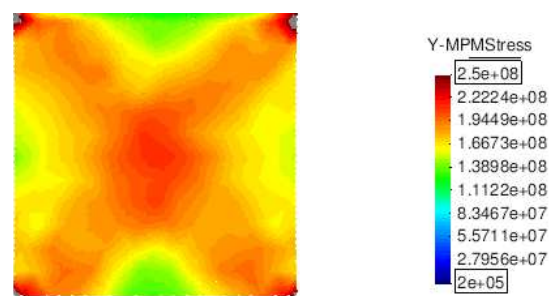

(b) $u$ - $p$ formulation

Figure 11 Tension test. Compressible case: Cauchy stress along loading axis. Black contour colour should be intended as out of range.

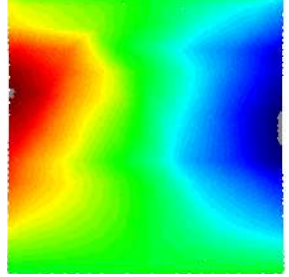

(a) $u$ formulation
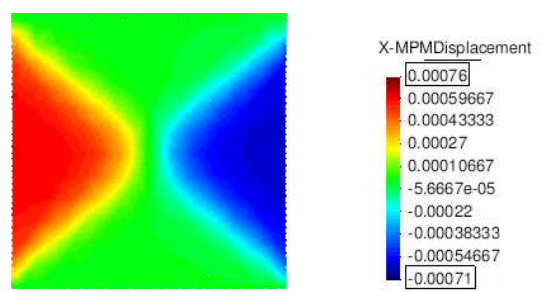

(b) $u$ - $p$ formulation

Figure 12 Tension test. Incompressible case: horizontal displacement
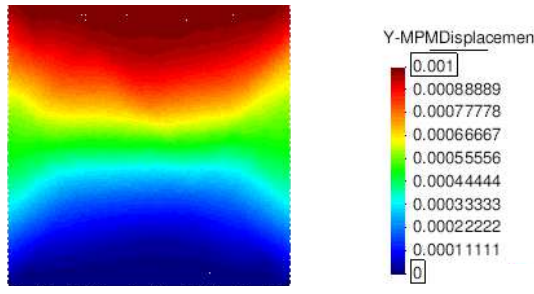

(a) $u$ formulation
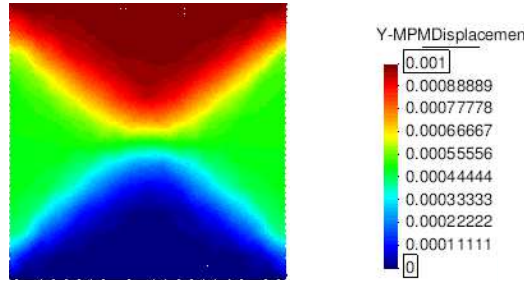

(b) $u$ - $p$ formulation

Figure 13 Tension test. Incompressible case: vertical displacement

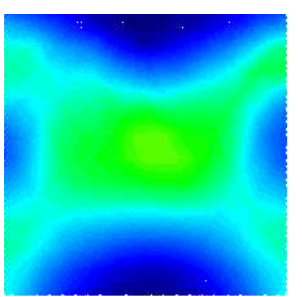

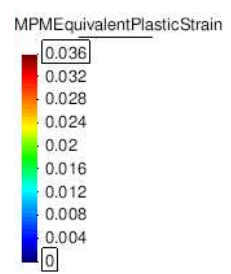

(a) $u$ formulation

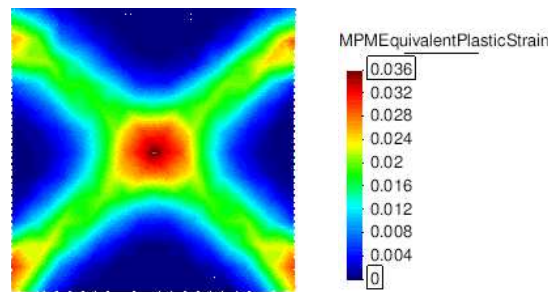

(b) $u$ - $p$ formulation

Figure 14 Tension test. Incompressible case: equivalent plastic strain 


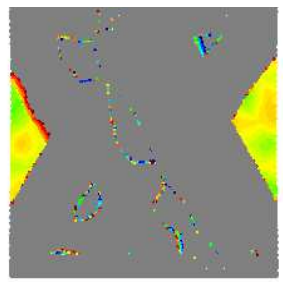

(a) $u$ formulation
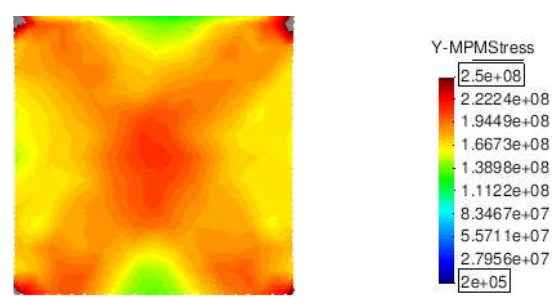

(b) $u$ - $p$ formulation

Figure 15 Tension test. Incompressible case: Cauchy stress along loading axis. Black contour colour should be intended as out of range.

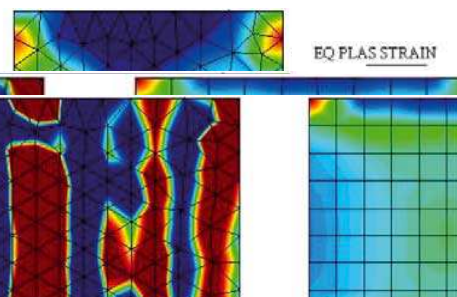

(a) equivalent plastic strain

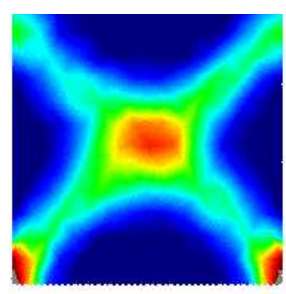

(c) equivalent plastic strain

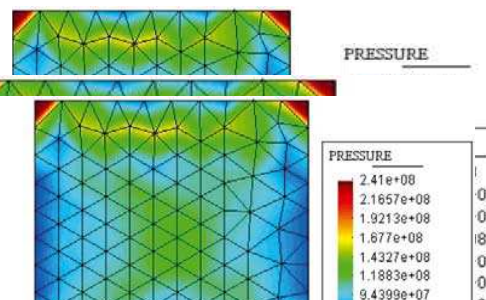

(b) pressure

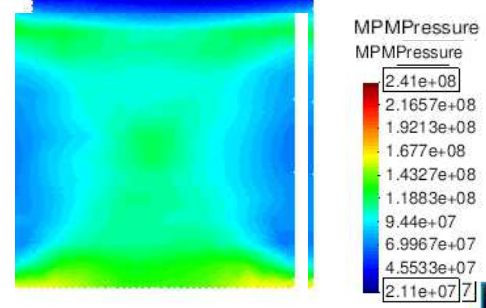

(d) pressure

Figure 16 Tension test. Incompressible case: results evaluated at a total imposed vertical displacement of $0.0001 \mathrm{~m}$. a) and b) Results in terms of equivalent plastic strain and pressure using a T1/P1 $u$ - $p$ formulation, taken from [16. c) and d) Results in terms of equivalent plastic strain and pressure evaluated with the MPM $\boldsymbol{u}$ - $p$ formulation presented in the current study.

\subsection{Plain strain rigid footing on undrained soil}

The last example is a plain strain rigid strip footing for the evaluation of the bearing capacity of the soil in undrained conditions, underneath the foundation. The soil is modelled as a purely cohesive weightless elasticperfectly plastic Mohr-Coulomb material with associative flow rule. For its implementation the return mapping defined in [46] has been followed and 


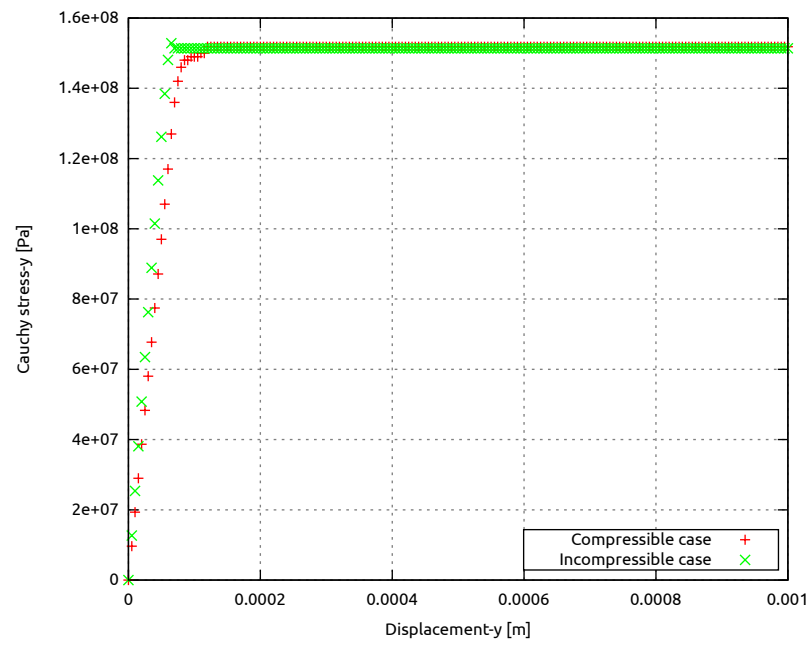

Figure 17 Tension test. Stress-Displacement curve. Comparison between the compressible case (red curve) and the incompressible curve (green curve).

the algorithm has been adapted to the case of finite strains plasticity [47,63, as briefly discussed in Section 2.3. The geometry, the boundary conditions and material properties are represented in Figure 18, where for symmetry only half of the domain is considered.
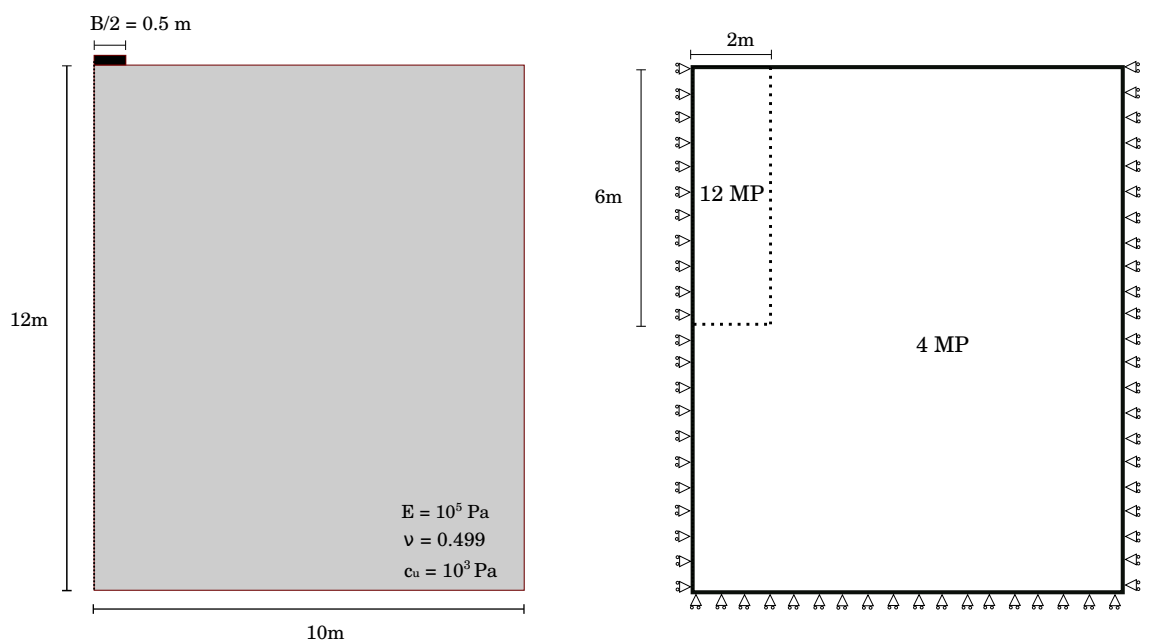

Figure 18 Rigid strip footing. Geometry, material properties, boundary conditions and initial material points density. 12 material points (MP) per element are used in the vicinity of the footing while only 4 are used in the rest of the domain. 
In the geomechanics community this is a classical benchmark for the validation of the constitutive law and of the numerical method adopted for its simulation. In the literature, the rigid strip footing has been studied by many authors. In 64] Nazem and coworkers solved this example in three different kinematics frameworks: a Total Lagrangian (TL), an Updated Lagrangian (UL) and an Arbitrary Lagrangian Eulerian (ALE) Finite Element Methods. They show that for high deformations an ALE method is more suitable than UL and TL strategies, avoiding mesh distortion with a remeshing technique. Even if the remeshing could smear a stress concentration and compromise the strain localization, they found that the load-displacement curve is comparable with the numerical solutions available in the literature. In 65 the technique of 64 is generalized to the case of higher order elements. The same test example has been also used to prove that the MPM represents an ideal numerical approach since it naturally tracks large deformations without the need of remeshing procedures. For instance, in [53] this example is successfully solved exploiting the capability of MPM to track large deformation and large displacement of the solid. However, the work of [53] is limited to the infinitesimal strain assumption.

In the current paper the work of [53] is generalized considering a stabilized mixed formulation, valid under geometric and material non-linearities. The simulation is performed using displacement control with steps of incremental vertical displacement $\Delta \boldsymbol{u}=-0.001 \mathrm{~m}$. The total displacement has been imposed in 2000 time steps which corresponds to twice the foundation width $B$. The discretization of the computational domain is performed through a unstructured triangular background mesh with a mesh size of $0.05 \mathrm{~m}$. At the interface between the foundation and the soil, where the largest deformations take place, a higher initial number of material points per element is used for a better resolution of the results (Figure 18).

In Figures 19, 20 and 21 the displacement and stress fields obtained with the $\boldsymbol{u}$ and $\boldsymbol{u}-\boldsymbol{p}$ formulations are compared. As expected, more reliable and accurate results are computed with the mixed finite element formulation. It can be noted that the final deformation is accurately described and an improvement is registered if the final deformation is compared with the numerical results of [64] and 65] which are more similar to the final configuration obtained through the displacement-based formulation. The need for a mixed formulation is evident when evaluating the vertical stress field. In Figure 21(a) the displacement-based formulation fails to evaluate a reliable stress response, as the magnitude of the vertical Cauchy stress is out of the expected range in the area where the foundation buries itself. On the other hand, the mixed formulation is able to evaluate a continuous stress field and using such result it is possible to evaluate the normalised load-displacement response of the foundation, which is used for the validation of the current example. 


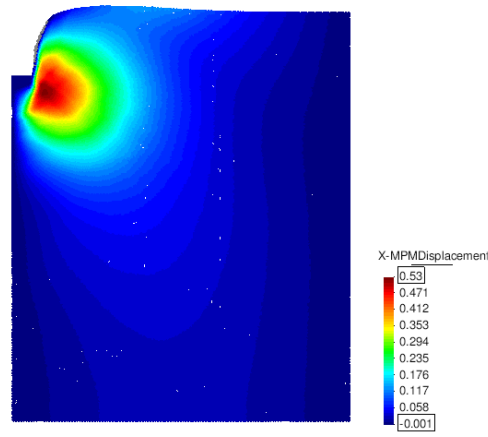

(a) $\boldsymbol{u}$ formulation

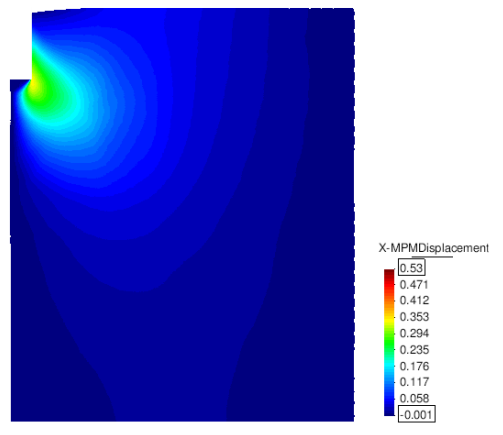

(b) $u$ - $p$ formulation

Figure 19 Rigid strip footing. Horizontal displacement

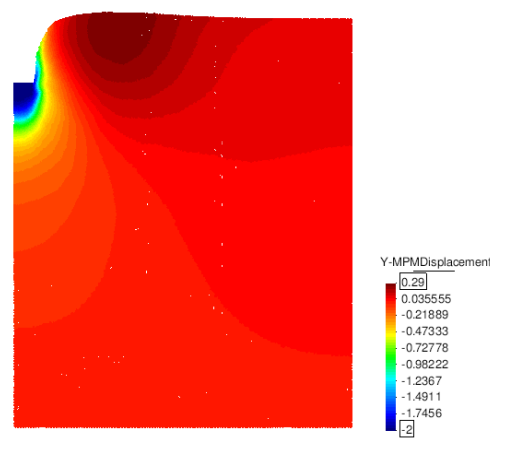

(a) $\boldsymbol{u}$ formulation

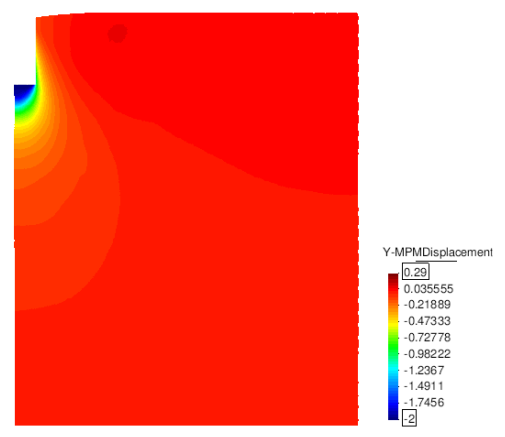

(b) $u$ - $p$ formulation

Figure 20 Rigid strip footing. Vertical displacement

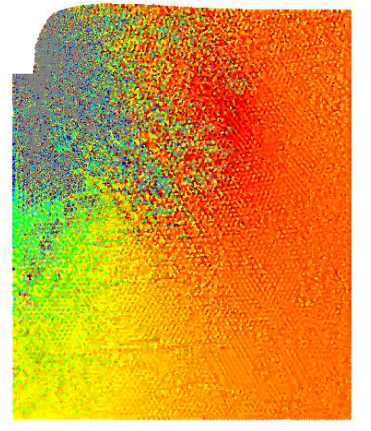

(a) $\boldsymbol{u}$ formulation

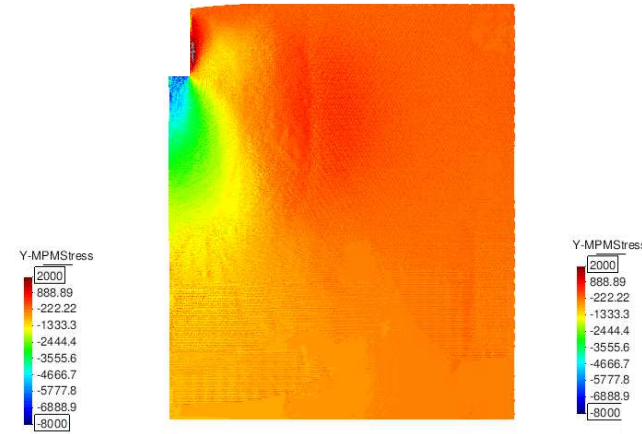

(b) $u$-p formulation

Figure 21 Rigid strip footing. Vertical Cauchy stress 
Since the problem has no analytical solution, the numerical result of [66], obtained through a sequential limit analysis formulation, is taken as reference solution. The problem is solved under the assumption of large deformations, hence, the bearing capacity of the soil is expected to be higher than the value of $2+\pi$ which corresponds to the small deformation case for a given footing displacement. Under this hypothesis, the mobilized soil resistance does not reach an asymptotic value, but gradually increases, as explained in [66]. In Figure 22, the result obtained through the $\boldsymbol{u}-\boldsymbol{p}$ formulation in terms of normalized bearing capacity of the soil, as a function of the normalized settlement, is depicted and compared with the benchmark solution. It can be observed that, the obtained curve is in good agreement with the reference solution [66]. The discrepancy that is observed for the initial values of the settlement is the consequence of the chosen material elastic properties. The Young Modulus $E$ and the Poisson's ratio $\nu$ have values which correspond to an undrained bulk modulus of $K_{u}=3,33 \cdot 10^{5} \mathrm{~Pa}$, which gives a ratio $K_{u} / c_{u}=3,33 \cdot 10^{3}$. In [53], the influence of this ratio on the normalised load-displacement curve is studied: the elastic response of the soil becomes less or more important and the bearing capacity of the soil can increase or decrease, for higher or lower values of this ratio, respectively. For this reason the numerical results plotted in Figure 22 have an important elastic response and are deviating during the initial phase of the simulation from the perfectly rigid behaviour of the benchmark solution.

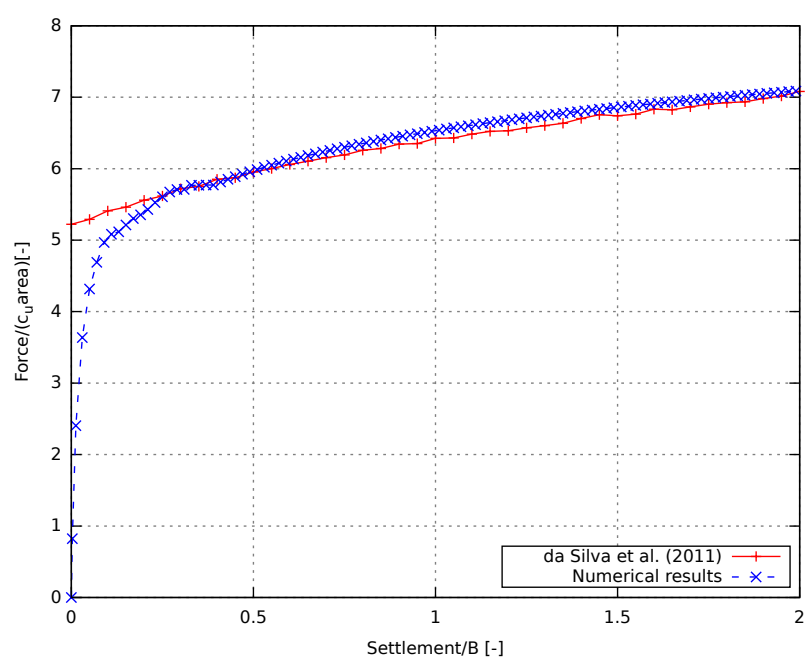

Figure 22 Rigid strip footing. Normalised load-displacement curve: comparison between reference solution taken from [66] and the $u$ - $p$ formulation solution presented in this work.

The example of the rigid footing on undrained soil has been validated using a stabilized mixed MPM formulation. The soil bearing capacity is 
well predicted and comparable with accurate numerical results from the literature. Moreover, a good description of the final deformation of the soil is achieved by using the MPM and its capability of solving large displacement and large deformation problems is equivalent, if not superior, to other techniques proposed in the literature [64,65].

\section{Conclusions}

In this paper a stabilized mixed formulation is presented within the framework of an implicit Material Point Method (MPM) for the solution of non-linear incompressible solid mechanics problems. The MPM is a particle method able to overcome typical issues of standard FEM when the continuum needs to undergo severe deformations, such as element tangling and extreme mesh distortion. The stabilized mixed $\boldsymbol{u}-\boldsymbol{p}$ formulation is tested through a series of benchmark examples and compared with a displacement-based $u$ approach. Firstly, the Cook's membrane problem, a bending dominated test, is investigated. Two cases, a compressible and a nearly-incompressible one, are solved through the $\boldsymbol{u}$ and $\boldsymbol{u}$ - $p$ strategies. It is demonstrated that the $\boldsymbol{u}-\boldsymbol{p}$ formulation always gives the best performance in term of convergence. In the quasi-incompressible case the volumetric locking issue is overcome and pressure oscillations are avoided if a stabilization term is added to the mixed finite element formulation. In the second example, a J2 plastic plate, subjected to uniform tension on one side and fixed to the other side, is simulated using both methodologies under an isochoric plastic flow condition. Comparing the displacement-based and mixed approach it is shown that better results are obtained through the $\boldsymbol{u}$ - $p$ strategy. Indeed, a more accurate definition of displacement, equivalent plastic strains and vertical Cauchy stress fields and a good agreement with the reference solution are observed. Despite volumetric locking issue is fixed in the case of the $\boldsymbol{u}-\boldsymbol{p}$ formulation, further problems, such as, mesh independence and strain localization, are not addressed in the current work and they represent interesting topics for a future research. As third benchmark test, the rigid footing on undrained soil is considered. This is a clear example, where the MPM represents a successful solution over the standard FEM since for the tracking of severe deformations the employment of remeshing procedures is avoided. In this case the good performances of the MPM $\boldsymbol{u}-p$ formulation are tested also under the finite deformation regime: a higher accuracy of the displacement and stress fields are confirmed. Moreover, evaluating the bearing capacity as function of the footing displacement, the load-displacement curve is obtained and used as validation tool to be compared with a reference solution. The numerical example shows that the MPM $\boldsymbol{u}-\boldsymbol{p}$ formulation is able to evaluate more accurate results in terms of displacement and stress fields, not only under near-incompressible state, avoiding the typical drawback of volumetric locking, but even under compressible conditions. 
In conclusion, the implicit MPM is a numerical strategy, able to successfully model highly deforming materials which may undergo incompressible or nearly-incompressible conditions.

Acknowledgements The research was supported by the Research Executive Agency through the T-MAPPP project (FP7 PEOPLE 2013 ITN-G.A.n607453). The Spanish Ministry of Economy and Competitiveness (Ministerio de Economía y Competitividad, MINECO) through the projects HIRMA (RTC-2016-4967-5), PRECISE (BIA2017-83805-R) is also greatly acknowledged. Finally Dr. Larese gratefully acknowledges the support of the Spanish ministry through her Juan de la Cierva Incorporacion (IJCI-2015-26484).

\section{References}

1. O. Zienkiewicz, R. Taylor, J. Zhu (eds.), The Finite Element Method: Its Basis and Fundamentals, seventh edition edn. (Butterworth-Heinemann, Oxford, 2013)

2. E. de Souza Neto, D. Perić, D. Owen, Computational Methods for Plasticity (John Wiley \& Sons, Ltd, 2008)

3. J.C. Simo, M.S. Rifai, A class of mixed assumed strain methods and the method of incompatible modes, International Journal for Numerical Methods in Engineering 29(8), 1595 (1990)

4. T.J.R. Hughes, Generalization of selective integration procedures to anisotropic and nonlinear media, International Journal for Numerical Methods in Engineering 15(9), 1413 (1980)

5. R.L. Taylor, P.J. Beresford, E.L. Wilson, A non-conforming element for stress analysis, International Journal for Numerical Methods in Engineering 10(6), 1211 (1976)

6. F. Auricchio, L.B. da Veiga, C. Lovadina, A. Reali, An analysis of some mixedenhanced finite element for plane linear elasticity, Computer Methods in Applied Mechanics and Engineering 194(27), 2947 (2005)

7. E. de Souza Neto, D. Perič, M. Dutko, D. Owen, Design of simple low order finite elements for large strain analysis of nearly incompressible solids, International Journal of Solids and Structures 33(20), 3277 (1996)

8. B. Moran, M. Ortiz, C.F. Shih, Formulation of implicit finite element methods for multiplicative finite deformation plasticity, International Journal for Numerical Methods in Engineering 29(3), 483 (1990)

9. J.C. Simo, F. Armero, Geometrically non-linear enhanced strain mixed methods and the method of incompatible modes, International Journal for Numerical Methods in Engineering 33(7), 1413 (1992)

10. B. Reddy, J. Simo, Stability and convergence of a class of enhanced strain methods, SIAM Journal on Numerical Analysis 32, 1705 (1995)

11. A. Ortiz-Bernardin, J. Hale, C. Cyron, Volume-averaged nodal projection method for nearly-incompressible elasticity using meshfree and bubble basis functions, Comput. Methods Appl. Mech. Engrg 285, 427 (2015)

12. T. Sussman, K.J. Bathe, A finite element formulation for nonlinear incompressible elastic and inelastic analysis, Computers \& Structures 26(1), 357 (1987) 
13. U. Brink, E. Stein, On some mixed finite element methods for incompressible and nearly incompressible finite elasticity, Computational Mechanics 19(1), 105 (1996)

14. M. Chiumenti, Q. Valverde, C.A. de Saracibar, M. Cervera, A stabilized formulation for incompressible elasticity using linear displacement and pressure interpolations, Computer Methods in Applied Mechanics and Engineering 191(46), 5253 (2002)

15. M. Cervera, M. Chiumenti, Q. Valverde, C.A. de Saracibar, Mixed linear/linear simplicial elements for incompressible elasticity and plasticity, Computer Methods in Applied Mechanics and Engineering 192(49), 5249 (2003)

16. M. Chiumenti, Q. Valverde, C.A. de Saracibar, M. Cervera, A stabilized formulation for incompressible plasticity using linear triangles and tetrahedra, International Journal of Plasticity 20(8), 1487 (2004)

17. M. Cervera, M. Chiumenti, R. Codina, Mixed stabilized finite element methods in nonlinear solid mechanics: Part ii: Strain localization, Computer Methods in Applied Mechanics and Engineering 199(37), 2571 (2010)

18. M. Cervera, M. Chiumenti, L. Benedetti, R. Codina, Mixed stabilized finite element methods in nonlinear solid mechanics. part iii: Compressible and incompressible plasticity, Computer Methods in Applied Mechanics and Engineering 285, 752 (2015)

19. J. Simo, R. Taylor, K. Pister, Variational and projection methods for the volume constraint in finite deformation elasto-plasticity, Computer Methods in Applied Mechanics and Engineering 51(1), 177 (1985)

20. F. Brezzi, On the existence, uniqueness and approximation of saddle-point problems arising from lagrangian multipliers, ESAIM: Mathematical Modelling and Numerical Analysis 8(R2), 129 (1974)

21. I. Babuška, The finite element method with lagrangian multipliers., Numerische Mathematik 20, $179(1972 / 73)$

22. I. Babuška, The finite element method with penalty., Mathematics of Computation 27, 221 (1973)

23. Fortin, Michel, An analysis of the convergence of mixed finite element methods, RAIRO. Anal. numér. 11(4), 341 (1977)

24. T.J. Hughes, L.P. Franca, M. Balestra, A new finite element formulation for computational fluid dynamics: V. circumventing the babu Åąka-brezzi condition: a stable petrov-galerkin formulation of the stokes problem accommodating equal-order interpolations, Computer Methods in Applied Mechanics and Engineering 59(1), 85 (1986)

25. T. Hughes, L. Franca, G. Hulbert, A new finite element formulation for computational fluid dynamics: Viii. the galerkin/least-squares method for advective-diffusive equations, Computer Methods in Applied Mechanics and Engineering 73(2), 173 (1989)

26. T.J. Hughes, Multiscale phenomena: Green's functions, the dirichlet-toneumann formulation, subgrid scale models, bubbles and the origins of stabilized methods, Computer Methods in Applied Mechanics and Engineering 127(1), 387 (1995)

27. E. Oñate, Derivation of stabilized equations for numerical solution of advective-diffusive transport and fluid flow problems, Computer Methods in Applied Mechanics and Engineering 151(1), 233 (1998). Containing papers presented at the Symposium on Advances in Computational Mechanics 
28. E. Oñate, A stabilized finite element method for incompressible viscous flows using a finite increment calculus formulation, Computer Methods in Applied Mechanics and Engineering 182(3), 355 (2000)

29. R. Codina, Stabilization of incompressibility and convection through orthogonal sub-scales in finite element methods, Computer Methods in Applied Mechanics and Engineering 190(13), 1579 (2000)

30. R. Codina, J. Blasco, Stabilized finite element method for the transient navier stokes equations based on a pressure gradient projection, Computer Methods in Applied Mechanics and Engineering 182(3), 277 (2000)

31. R. Codina, Stabilized finite element approximation of transient incompressible flows using orthogonal subscales, Computer Methods in Applied Mechanics and Engineering 191(39), 4295 (2002)

32. C. Mast, P. Mackenzie-Helnwein, P. Arduino, G. Miller, W. Shin, Mitigating kinematic locking in the material point method, Journal of Computational Physics 231(16), 5351 (2012)

33. S. Kularathna, K. Soga, Implicit formulation of material point method for analysis of incompressible materials, Computer Methods in Applied Mechanics and Engineering 313, 673 (2017)

34. A.J. Chorin, Numerical solution of the navier-stokes equations, Mathematics of computation 22(104), 745 (1968)

35. F. Zhang, X. Zhang, K.Y. Sze, Y. Lian, Y. Liu, Incompressible material point method for free surface flow, Journal of Computational Physics 330, 92 (2017)

36. I. Iaconeta, A. Larese, R. Rossi, E. Oñate, An implicit material point method applied to granular flows, Procedia Engineering 175, 226 (2017). Proceedings of the 1st International Conference on the Material Point Method (MPM 2017)

37. P. Dadvand, A framework for developing finite element codes for multidisciplinary applications. (PhD thesis: Universidad Politécnica de Cataluña, 2007)

38. P. Dadvand, R. Rossi, E. Oñate, An object-oriented environment for developing finite element codes for multi-disciplinary applications, Archives of Computational Methods in Engineering 17, 253 (2010)

39. I. Iaconeta, A. Larese, R. Rossi, Z. Guo, Comparison of a material point method and a galerkin meshfree method for the simulation of cohesivefrictional materials., Materials 1010 (2017)

40. C.R. Dohrmann, P.B. Bochev, A stabilized finite element method for the stokes problem based on polynomial pressure projections, International Journal for Numerical Methods in Fluids 46(2), 183 (2004)

41. J. Rodriguez, J. Carbonell, J. Cante, J. Oliver, The particle finite element method (pfem) in thermo-mechanical problems, International Journal for Numerical Methods in Engineering (2015)

42. L. Monforte, J.M. Carbonell, M. Arroyo, A. Gens, Performance of mixed formulations for the particle finite element method in soil mechanics problems, Computational Particle Mechanics pp. 1-16 (2016)

43. P. Wriggers, Computational Contact Mechanics (Springer, 2006)

44. J. Simo, T. Hughes, Computational Inelasticity (Springer-Verlag New York, 1998)

45. J.C. Simo, A framework for finite strain elastoplasticity based on maximum plastic dissipation and the multiplicative decomposition: Part i. continuum formulation, Computer methods in applied mechanics and engineering 66(2), 199 (1988) 
46. J. Clausen, L. Damkilde, L. Andersen, Efficient return algorithms for associated plasticity with multiple yield planes, International Journal for Numerical Methods in Engineering 66(6), 1036 (2006)

47. J. Simo, Algorithms for static and dynamic multiplicative plasticity that preserve the classical return mapping schemes of the infinitesimal theory, Computer Methods in Applied Mechanics and Engineering 99(1), 61 (1992)

48. J. Simo, Numerical analysis and simulation of plasticity, Handbook of Numerical Analysis 6, 183 (1998)

49. F. Harlow, The particle-in-cell computing method for fluid dynamics, Methods for Computational Physics 3, 319 (1964)

50. D. Sulsky, Z. Chen, H. Schreyer, A particle method for history-dependent materials, Computer Methods in Applied Mechanics and Engineering 118(12), 179 (1994)

51. D. Sulsky, S.J. Zhou, H.L. Schreyer, Application of a particle-in-cell method to solid mechanics, Computer Physics Communications 87(1-2), 236 (1995)

52. Z. Wieckowski, The material point method in large strain engineering problems, Computer Methods in Applied Mechanics and Engineering 193(39-41), 4417 (2004)

53. W. Sołowski, S. Sloan, Evaluation of material point method for use in geotechnics, International Journal for Numerical and Analytical Methods in Geomechanics 39(7), 685 (2015)

54. S. Bardenhagen, E. Kober, The generalized interpolation material point method, CMES - Computer Modeling in Engineering and Sciences 5(6), 477 (2004)

55. A. Sadeghirad, R. Brannon, J. Burghardt, A convected particle domain interpolation technique to extend applicability of the material point method for problems involving massive deformations, Int. J. Numer. Meth. Eng. 86(12), 1435 (2011)

56. A. Sadeghirad, R. Brannon, J. Guilkey, Second-order convected particle domain interpolation (cpdi2) with enrichment for weak discontinuities at material interfaces, International Journal for Numerical Methods in Engineering 95(11), 928 (2013)

57. M. Steffen, P. Wallstedt, J. Guilkey, R. Kirby, M. Berzins, Examination and analysis of implementation choices within the material point method (mpm), Computer Modeling in Engineering and Sciences 31(2), 107 (2008)

58. M. Steffen, R.M. Kirby, M. Berzins, Analysis and reduction of quadrature errors in the material point method $(\mathrm{mpm})$, International journal for numerical methods in engineering $\mathbf{7 6}(6), 922$ (2008)

59. Y.G. Motlagh, W.M. Coombs, An implicit high-order material point method, Procedia Engineering 175, 8 (2017). Proceedings of the 1st International Conference on the Material Point Method (MPM 2017)

60. R. Cook, Improved Two-Dimensional Finite ELement, Journal of the Structural Division 100, 1851 (1974)

61. A. Franci, Unified Lagrangian formulation for fluid and solid mechanics, fluidstructure interaction and coupled thermal problems using the PFEM (PhD thesis: Universitat Politécnica de Catalunya, 2015)

62. M. Cervera, M. Chiumenti, R. Codina, Mixed stabilized finite element methods in nonlinear solid mechanics: Part i: Formulation, Computer Methods in Applied Mechanics and Engineering 199(37), 2559 (2010) 
63. R.I. Borja, K.M. Sama, P.F. Sanz, On the numerical integration of threeinvariant elastoplastic constitutive models, Computer Methods in Applied Mechanics and Engineering 192(9), 1227 (2003)

64. M. Nazem, D. Sheng, J.P. Carter, Stress integration and mesh refinement for large deformation in geomechanics, International Journal for Numerical Methods in Engineering 65(7), 1002 (2006)

65. M. Kardani, M. Nazem, J. Carter, A. Abbo, Efficiency of high-order elements in large-deformation problems of geomechanics, International Journal of Geomechanics 15(6), 04014101 (2014)

66. M.D. Silva, K. Krabbenhoft, A. Lyamin, S. Sloan, Rigid-plastic largedeformation analysis of geotechnical penetration problems, Proceeding of the 13th IACMAG Conference. Computer methods for geomechanics: frontiers and new applications 1, 42 (2011) 